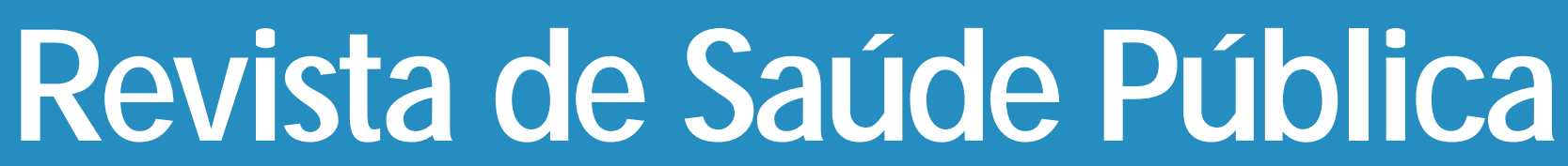

$\begin{array}{lllllll}J & 0 & \mathbf{U} & \mathbf{R} & \mathbf{N} & \mathbf{A} & \mathbf{L}\end{array}$

0 F

$\mathbf{P} \mathbf{U}$

B L I C

H E A L T H

\title{
Tétano acidental: análise do perfil clínico e epidemiológico de casos internados em hospital universitário*
}

\section{Acquired tetanus: clinical and epidemiological characteristics of patients in an university hospital}

Vera M. S. F. Lima, Márcia T. G arcia, Mariângela R. Resende, Simone A. N ouer, Eliane 0 . M. Campos, Priscila M. O. Papaiordanou e Luiz J. da Silva

Núcleo de Vigilância Epidemiológica do Hospital das Clínicas da Universidade Estadual de Campinas (UNICAMP). Campinas, SP - Brasil (M.R.R., S.A.N., E.O.M.C.), Departamento de Clínica Médica da Faculdade de Ciências Médicas da U NICAMP. Campinas, SP - Brasil (V.M.S.F.L., M.T.G., P.M.O.P. e L.J.S.) 


\title{
Tétano acidental: análise do perfil clínico e epidemiológico de casos internados em hospital universitário*
}

\section{Acquired tetanus: clinical and epidemiological characteristics of patients in an university hospital}

\author{
Vera M. S. F. Lima, Márcia T. G arcia, Mariângela R. Resende, Simone A. N ouer, Eliane 0. \\ M. Campos, Priscila M. O. Papaiordanou e Luiz J. da Silva \\ Núcleo de Vigilância Epidemiológica do Hospital das Clínicas da Universidade Estadual de \\ Campinas (U NICAMP). Campinas, SP - Brasil (M.R.R., S.A.N., E.O.M.C.), Departamento de Clínica \\ Médica da Faculdade de Ciências M édicas da UNICAM P. Campinas, SP - Brasil (V.M.S.F.L., M.T.G., \\ P.M.O.P. e L.J.S.)
}

\begin{abstract}
Resumo
Introdução

O número de casos notificados de tétano acidental no Estado de São Paulo sofreu redução. O declínio do número de casos de qualquer doença sempre traz transformações no seu perfil epidemiológico, que devem sempre ser analisadas para aprimorar as medidas preventivas. Assim, foi analisado o perfil clínico e epidemiológico dos casos de tétano internados em hospital universitário de Campinas de 1989 a 1996.

Material e Método

Estudo descritivo e retrospectivo (série de casos). Todos os pacientes com diagnóstico de tétano de janeiro de 1989 a março de 1996, internados no hospital universitário, foram analisados.

Resultados Catorze (28\%) eram da zona rural e $36(72 \%)$ da zona urbana. A idade média foi de 47,6 anos e a mediana de 49,5. Dos pacientes da zona rural, 42,8\% tinham até 30 anos e $21,42 \%$ tinham mais de 50 anos, sendo a média 36,21 e a mediana 34,5; dos pacientes da zona urbana, 13,9\% tinham até 30 anos e 58,3\% mais de 50 anos, sendo a média de 52,2 e a mediana de 54,5. A letalidade foi de $20 \%$, mais elevada nos pacientes curarizados $(60 \%)$.

Conclusão

Na região estudada existem dois padrões epidemiológicos: o rural, com maior número de jovens, refletindo uma vacinação inadequada, e o urbano, semelhante ao dos países desenvolvidos, com predomínio das faixas etárias mais altas.
\end{abstract}

Tétano, epidemiologia. Diagnóstico clínico.

\footnotetext{
* Apresentado na forma de comunicação no Infecto 96 - IV Congresso da Sociedade Brasileira de Infectologia, Recife, 1996. Correspondência para/Correspondence to: Luiz J. da Silva - Cx. Postal 6019 - UNICAMP - 13081-970 Campinas, SP - Brasil. E-mail: luisjs@guttenberg.correionet.com.br

Edição subvencionada pela FAPESP (Processo no 97/09815-2).

Recebido em 4.12.1996. Reapresentado em 19.5.1997. Aprovado em 21.10.1997.
} 


\begin{abstract}
Introduction

Notwithstanding its substantial decline over the last two decades, acquired tetanus is still a serious health problem in most developing countries. Epidemiological transition is often cited as an explanation for this decline, the increase in vaccination coverage of children being the most obvious cause. Few studies have looked carefully at the current epidemiological patterns of acquired tetanus in developing countries.

Methodology

A descriptive, retrospective (series of cases). An acute care 400 - bed university referral hospital situated in a densely populated and highly urbanized area in Southeastern Brazil (Campinas, SP). Patient records the data-base analysed were from the Epidemiological Surveillance Unit of the hospital.

Results $\quad$ In the 57 month period from January 1989 to March 1996 fifty-three patients were admitted with a diagnosis of acquired tetanus. Fifty patients had clinical confirmation, 3 were otherwise diagnosed. Thirty-two (64\%) were male and 18 (36\%) female. Fourteen (28\%) were from rural areas and $36(72 \%)$ from urban. Mean age was 47.6 years, with a median of 49.5 . Of the rural patients, $42.85 \%$ were under 30 years and $21.42 \%$ were over 50, mean age was 36.21 with a median of 34.5. Fewer urban patients were under 30 (13.88\%) than over 50 (58.33\%), mean age was 52.19 with a median of 54.5. Trismus was the most frequent $(92.0 \%)$ clinical sign on admittance, followed by abdominal muscular rigidity (84.0\%). Treatment measures were uniform and included tetanus immune globulin, antibiotics, surgical debridement of the wound when feasible, diazepan or curare depending on the intensity of spasms. In the second half of the study period, penicillin was replaced by metronidazol. Overall case fatality rate was $20 \%$, in patients that had to receive curare, it was $60 \%$. Hospitalization exceeded 21 days in 56\% (28) of the cases, only 10\% (5) had a hospital stay of less than 7 days.

Conclusion A high proportion of patients were from rural areas, despite an urbanization rate of more than $90 \%$. In the Campinas region there are two different epidemiological patterns of acquired tetanus: a rural pattern, with a higher proportion of young er patients, determined by an inadequate immunization rate and an urban pattern, similar to that found in industrialized countries, with a higher proportion of older patients.

Recommendations

There is an obvious need to immunize older individuals in urban areas and young adults in rural areas. The elimination of acquired tetanus will only be achieved with a wider and more intensive adult vaccination program.
\end{abstract}

Tetanus, epidemiology. Diagnosis, clinical.

\section{INTRO DUCCÃO}

Mais de um século após a demonstração de que sua profilaxia é possível, o tétano acidental continua um sério problema de saúde pública ${ }^{4,15}$. Nos países desenvolvidos sua ocorrência está restrita às faixas etárias mais altas ${ }^{16}$ e é apenas uma sombra do que foi nas primeiras décadas deste Século; no entanto, não dá mostras de desaparecimento. No Estado de São Paulo os casos notificados de tétano acidental apresentam tendência à redução $0^{10,11}$, ainda que esta não tenha sido tão significativa quanto seria de se esperar diante da expressiva cobertura vacinal, superior a $80 \%^{18}$. À primeira vista, esta tendência seria uma decorrência da transição epidemiológica em curso, tanto no Brasil como em outros países em desenvolvimento, principalmente quando se vê o virtual desaparecimento do tétano neonatal ${ }^{7}$. Seus determinantes seriam a ampliação da cobertura vacinal e a melhoria das condições de acesso e assistência dos serviços de saúde ${ }^{5}$ Não há dúvida de que estes dois fatos se verificaram nas duas últimas décadas $^{14,18}$, não obstante a recessão do investimento na área social por parte do governo. A transição epidemiológica é um fenômeno sempre lembrado, mas raramente analisado em detalhe. 
O total de casos de tétano em São Paulo passou de 559 em 1968 para 111 em 1995. Aparentemente, ou o ano de 1968 foi atípico ou se verificou um viés de notificação. Desconsiderando o ano de 1968, vêse que a queda foi bem menor: de $313 \mathrm{em} 1970$ para 111 em 1995, uma redução de 64,5\%. Essa redução é mais significativa se forem considerados apenas os casos de tétano neonatal. A notificação passou a ser discriminada apenas a partir de 1982, quando foram notificados 18 casos de tétano neonatal, em 1995 apenas 3, uma redução de $83,3 \%$, quando no mesmo período a redução dos casos de tétano acidental foi de apenas $11,9 \%^{10,11}$.

O declínio do número de casos de qualquer doença sempre traz transformações no seu perfil epidemiológico e que devem ser analisadas para aprimorar as medidas preventivas.

Mesmo nos países industrializados, apesar da redução na sua incidência, o tétano continua sendo uma realidade presente, com um considerável segmento populacional susceptível. Essa susceptibilidade não é homogeneamente distribuída, havendo um gradiente etário - a susceptibilidade aumenta com a idade - e social - é maior entre os menos favorecidos economicamente. Nesses países, o tétano ocorre quase que exclusivamente em pacientes com mais de 60 anos de idade. Possivelmente a reduzida ocorrência de casos de tétano nesses países se deva mais à qualidade da assistência médica quando dos ferimentos do que a uma elevada prevalência de títulos protetores de anticorpos. Recentes trabalhos mostraram que, não obstante, uma elevada cobertura vacinal em crianças, a população adulta norte-americana, particularmente aquela com maior dificuldade de acesso aos serviços de saúde, é susceptível ao tétano $2,4,15,16$.

As análises de séries de casos podem fornecer mais do que simplesmente esclarecimentos acerca das características clínicas, permitem uma visão do perfil epidemiológico e dos fatores prognósticos, permitindo medidas terapêuticas mais precisas para os próximos pacientes, como também propor medidas de controle da doença e uma melhor compreensão dos seus determinantes.

O objetivo do presente trabalho foi, pois, analisar o perfil clínico e epidemiológico dos casos de tétano internados no hospital universitário de Campinas, de 1989 a 1996 e inferir o padrão de ocorrência do tétano acidental na região de Campinas.

\section{CASU ÍSTICA E MÉTO DO}

Foram pesquisados todos os pacientes internados com diagnóstico de tétano (CID - 9: 037) no período de janeiro de 1989 a março de 1996. De um total de 53, foram excluídos 3 por ter o diagnóstico modificado após a internação. Não havia casos de tétano neonatal.

Trata-se de estudo do tipo descritivo e retrospectivo (série de casos).

Foram analisadas as fichas de notificação e dos prontuários dos pacientes internados no período, com diagnóstico de tétano acidental.

Os dados foram coletados do banco de dados do Núcleo de Vigilância Epidemiológica e dos prontuários da Divisão de Arquivo Médico, ambos do hospital estudado.

\section{RESU LTAD O $S$}

\section{Características D emográficas}

Dos 50 pacientes analisados, $32(54 \%)$ eram homens e 18 (46\%) mulheres; 14 (28\%) eram da zona rural e $36(72 \%)$ da zona urbana.

A idade média foi de 47,6 anos e a mediana de 49,5. A distribuição dos pacientes por faixa etária está na Tabela 1.

Dos pacientes da zona rural, 42,8\% tinham até

Tabela 1 - Distribuição dos pacientes com diagnóstico de tétano acidental, conforme a faixa etária e procedência.

Table 1 - Distribution of patients with diagnosis of accidental tetanus by age group and place of origin.

\begin{tabular}{lrrrrrrrrr}
\hline Procedência & \multicolumn{4}{c}{ Rural } & \multicolumn{3}{c}{ Urbana } & \multicolumn{2}{c}{ Rural + Urbana } \\
\hline $\begin{array}{l}\text { Faixa etária } \\
\text { (anos) }\end{array}$ & $\mathrm{n}$ & $\%$ & $\%$ acumul. & $\mathrm{n}$ & $\%$ & $\%$ acumul. & $\mathrm{n}$ & $\%$ & $\%$ acumul. \\
\hline $0-10$ & 1 & 7,1 & 7,1 & 1 & 2,8 & 2,8 & 2 & 4,0 & 4,0 \\
$11-20$ & 2 & 14,3 & 21,4 & 4 & 11,1 & 13,9 & 6 & 12,0 & 16,0 \\
$21-30$ & 3 & 21,4 & 42,8 & 0 & 0 & 13,9 & 3 & 6,0 & 22,0 \\
$31-40$ & 2 & 14,3 & 57,1 & 4 & 11,1 & 25,0 & 6 & 12,0 & 34,0 \\
$41-50$ & 2 & 14,3 & 71,4 & 7 & 19,4 & 44,4 & 9 & 18,0 & 52,0 \\
$51-60$ & 3 & 21,4 & 92,8 & 7 & 19,4 & 63,8 & 10 & 20,0 & 72,0 \\
$61-70$ & 1 & 7,1 & 99,9 & 3 & 8,3 & 72,1 & 4 & 8,0 & 80,0 \\
71 ou + & 0 & 0 & 99,9 & 10 & 27,8 & 99,9 & 10 & 20,0 & 100,0 \\
\hline Total & 14 & & 100,0 & 36 & & 100,0 & 50 & & 100,0 \\
\hline
\end{tabular}


30 anos de idade, e 28,6\% tinham mais de 50 anos; dos pacientes da zona urbana apenas $13,9 \%$ tinham até 30 anos e 55,5\% mais de 50. A idade média dos pacientes da zona rural foi de 36,2 anos, com uma mediana de 34,5 . Na zona urbana a idade média foi de 52,2 anos e a mediana de 54,5.

Com exeção de um, os pacientes eram procedentes de 29 diferentes municípios. Do total, 8 (16\%) eram de Campinas e 7 (14\%) dos municípios vizinhos de Sumaré e Hortolândia. A distribuição difere da média observada no hospital universitário, onde de 30 a $34 \%$ dos pacientes são provenientes de Campinas* e cerca de $20 \%$ dos municípios de Hortolândia e Sumaré, conjuntamente.

No mesmo período, a regional da vigilância epidemiológica da Secretaria Estadual da Saúde registrou 40 casos** de tétano.

\section{Q uadro Clínico e Tratamento}

Trismo foi a manifestação clínica mais comum na internação (92\%), seguido de rigidez abdominal (84\%). A provável porta de entrada está na Tabela 2.

O período de incubação foi de até 7 dias em 18 pacientes $(36,0 \%)$, de 8 a 14 em $15(30,0 \%)$ e de 15

Tabela 2 - Provável porta de entrada da infecção por tétano acidental em pacientes internados.

Table 2 - Probable site of entry by accidental tetanus.

\begin{tabular}{lrr}
\hline Tipo de ferimento & $\mathrm{n}$ & $\%$ \\
\hline Cirurgia & 2 & 4 \\
Cortante & 9 & 18 \\
Escoriação & 7 & 14 \\
Laceração & 11 & 22 \\
Punctório & 12 & 24 \\
Queimadura & 1 & 2 \\
Outros & 5 & 10 \\
Indeterminado & 3 & 6 \\
\hline Total & 50 & 100 \\
\hline
\end{tabular}

a 22 em $9(18,0 \%)$. Em $8(16,0 \%)$ pacientes não foi possível determinar o período de incubação. Os dados existentes nos prontuários não permitiram uma adequada análise dos pacientes conforme o período de progressão.

O tratamento foi uniforme e constituiu no uso de imunoglobulina humana específica, antibioticoterapia, debridamento do ferimento quando factível, diazepam ou curare conforme a gravidade, seguindo a tendência da literatura ${ }^{13}$. Por volta da metade do período, a penicilina foi substituída pelo metronidazol.
O tempo de internação foi maior do que 21 dias em 56\% (28) dos casos, apenas 10\% (5) tiveram internação de até 7 dias.

A letalidade foi de $20 \%$ (10), mais elevada nos pacientes curarizados (60\% [6]) e nos mais idosos. Nenhum dos que vieram a falecer havia recebido vacinação anterior.

A letalidade variou diretamente com a idade e inversamente com o período de progressão e de incubação.

\section{DISCU SSÃO}

Apesar da redução da incidência, o tétano acidental continua sendo um problema de saúde pública pela sua alta letalidade e tratamento custoso. Há uma alta proporção de pacientes da zona rural numa região onde a taxa de urbanização é superior a 90\%. Na região de Campinas parecem existir dois padrões epidemiológicos: o rural, com maior número de jovens, refletindo uma cobertura vacinal inadequada, e o urbano, semelhante ao dos países desenvolvidos, com predomínio das faixas etárias mais altas.

No período considerado, foram notificados 40 casos de tétano procedentes de diversos municípios da região. $\mathrm{O}$ fato deste número ser menor deve refletir subnotificação e alguns casos internados no hospital universitário que são de fora da área de abrangência da regional de saúde. Apesar da diferença de totais (Tabela 3), há uma estreita correlação entre os casos internados no hospital e a distribuição dos casos na região, o que permite fazer inferências acerca do comportamento do tétano na região de Campinas.

A idade média foi de 47,6 anos e a mediana de 49,5. Numa série de casos da Polônia ${ }^{1}$, com 30 casos considerados graves - 26 foram submetidos a ventilação assistida - a idade média foi de 64 anos, com uma letalidade de 53,3\%, semelhante à verificada no presente estudo, onde entre os pacientes curarizados, a letalidade foi de $60 \%$. A menor idade dos pacientes estudados talvez tenha influído. No entanto, pelo menos uma série de casos, da Suíça ${ }^{5}$, sugere que idade não é fator preditivo de letalidade, ao contrário do senso comum. A experiência norteamericana também mostra que o prognóstico dos pacientes com tétano acidental independe da idade ${ }^{6}$.

A letalidade é inferior à observada no Estado de São Paulo como um todo $(44,4 \%)$, no início do

* Dados inéditos

** Distrito Regional XII - Dados inéditos. 
Tabela 3 - Distribuição dos pacientes intertnados no hospital universitário de Campinas e dos casos de tétano acidental notificados à Diretoria Regional de Saúde 12 (Campinas), durante o período do estudo, conforme o município de ocorrência .

Table 3 - Distribution of patients admitted to the hospital and notified cases of acquired tetanus, according to the municipality of occurence.

\begin{tabular}{lcc}
\hline Procedência & $\begin{array}{c}\text { Internados } \\
\text { no hospital }\end{array}$ & $\begin{array}{c}\text { Notificados } \\
\text { DIR 12 }\end{array}$ \\
\hline Americana & 1 & 2 \\
Amparo & 1 & 1 \\
Andradas & 1 & 0 \\
Araras & 1 & 0 \\
Bragança Paulista & 1 & 1 \\
Caconde & 0 & 1 \\
Campinas & 8 & 9 \\
Capivari & 1 & 1 \\
Conchal & 1 & 0 \\
Cosmópolis & 1 & 1 \\
Elias Fausto & 1 & 1 \\
Holambra & 1 & 0 \\
Hortolândia & 2 & 2 \\
Indaiatuba & 3 & 2 \\
Itapira & 2 & 1 \\
Itu & 3 & 2 \\
Jaguariúna & 1 & 1 \\
Limeira & 1 & 1 \\
Louveira & 1 & 0 \\
Nova Odessa & 1 & 0 \\
Pirassununga & 2 & 2 \\
Porto Ferreira & 1 & 0 \\
Rio Claro & 1 & 1 \\
Salto & 1 & 0 \\
Sta. Bárbara D `O este & 2 & 2 \\
Sto. Antônio da Posse & 2 & 0 \\
São Pedro & 0 & 1 \\
Serra Negra & 1 & 1 \\
Socorro & 2 & 0 \\
Sumaré & 5 & 6 \\
Valinhos & 1 & 1 \\
\hline Total & 50 & 40 \\
\hline Fonte: Secretaria de Estado da Saúde. Diretoria Regional de Saúde & 12 \\
Caninas & &
\end{tabular}

Fonte: Secreta
Campinas.

período de estudo. É também inferior à de unidades especializadas no tratamento do tétano $(34,5 \%)$ e de unidades não especializadas $(49,5 \%)^{8}$.

Entendendo-se a transição epidemiológica como a passagem gradativa de um perfil nosológico de país subdesenvolvido para o de um país industrializado, a redução do número de casos em São Paulo e a média de idade dos casos situada numa faixa intermediária entre países como a Indonésia e os Estados Unidos, pareceria um reflexo desta transição. Esta impressão fica reforçada quando se analisa a evolução da distribuição etária proporcional dos casos de tétano (acidental e neonatal) no Brasil entre 1979 e 1989 (Litvoc ${ }^{8,}$ 1991).

A ampliação da cobertura vacinal traria uma gradativa elevação da idade média do grupo não vacinado, e portanto, susceptível. Na realidade, ainda que a ampliação da cobertura vacinal seja paralela à redução do segmento susceptível, este parece ser muito maior do que inicialmente se imaginava. A persistência da ocorrência de casos mesmo em países industrializados e os dados de soroprevalência de anticorpos contra o tétano mostram que a susceptibilidade ao tétano é muito mais prevalente do que se poderia inferir pela ocorrência de casos clínicos.

O quadro clínico e a evolução dos pacientes não diferem do relatado na literatura contemporânea. O tratamento seguiu o consensual. No momento presente não existem controvérsias importantes no tratamento do tétano ${ }^{17} \mathrm{e}$ este não parece ter sido fator determinante na letalidade nesta série, ao contrário da idade e do período de progressão. O objetivo não foi o de analisar o tratamento, uma vez que o delineamento do presente estudo não se presta a tanto. A letalidade verificada nas diferentes séries de casos publicados parece depender mais da distribuição etária dos pacientes e das condições de tratamento intensivo $0^{6,17}$.

Os gradientes geográfico e social não são exclusivos dos países em desenvolvimento. A cobertura costuma ser menor em imigrantes e migrantes, não só pelo padrão econômico geralmente menos favorecido, como pela maior mobilidade geográfica, o que prejudica a ação dos programas sociais, como saúde e educação $0^{2,412}$.

A incidência proporcionalmente maior na zona rural é talvez reflexo de uma menor cobertura vacinal associada a um risco aumentado de exposição. Esta não parece ser uma característica regional. Mesmo nos países desenvolvidos, uma cobertura vacinal reduzida está associada à procedência da zona rural ${ }^{2}$, o que sem dúvida deve levar a uma maior incidência do tétano. Uma percentagem significativa dos casos de tétano na Finlândia está associada com profissões ligadas à zona rural, como agricultura, reflorestamento e pecuária9.

Ainda há necessidade de se ampliar a cobertura vacinal de jovens na zona rural e de adultos na zona urbana. A eliminação do tétano acidental só se dará com uma maior atenção à população idosa e à rural. Os achados clínicos e resultados de tratamento estão de acordo com o encontrado na literatura, assinalando-se que os resultados parecem ser melhores do que os do restante do Estado de São $\mathrm{Paulo}^{8}$, ainda que não seja possível estabelecer-se comparação devido às diferenças das casuísticas.

O tratamento do tétano acidental no hospital estudado segue o consenso da literatura e apresenta resultados que podem ser considerados acima da média em termos de sobrevida.

O tétano acidental continua sendo sério problema de saúde pública no Estado de São Paulo. A vacinação da população infantil não é suficiente para eliminar 
a doença. Na região de Campinas existem dois padrões epidemiológicos do tétano acidental:

- rural, com um importante contingente de jovens, refletindo não só o risco das atividades ligadas à agropecuária, como também uma insuficiente cobertura vacinal, sugerindo haver uma concentração de não vacinados no meio rural.

- urbano, com predomínio de idosos, refletindo a inadequada vacinação de adultos e uma assistência médica precária, principalmente quando se tem dois casos possivelmente iatrogênicos (ocorrência após procedimento cirúrgico).

A eliminação do tétano acidental exige a vacinação sistemática dos adultos e a adequada assistência médica aos ferimentos. A vacinação de adultos é mais complexa pois a maioria não procura regularmente serviços de saúde. No entanto, existem várias oportunidades que são perdidas:

- programas de atenção a enfermidades crônicas, como diabetes e hipertensão,

- programas de controle de câncer ginecológico,

- consultas eventuais, particularmente em serviços de urgência ou de pronto-atendimento.

É fundamental que os serviços públicos de saúde deixem de repousar sobre os louros da vitória que foi a elevada cobertura vacinal na infância e se dediquem à tarefa árdua de efetivamente eliminar o tétano.

\section{REFERÊNCIAS}

1. CABAN, J. \& POSTAWA, B. Chorzy na tezec o przebiegu ciezkim leczeni w klinice chorob zakaznych w latach 19861988. Przegl. Lek., 47: 633-6, 1990.

2. ELNICKI, D. M.; MORRIS, D. K.; SHOCKCOR, W. T. Patient-perceived barriers to preventive health care among indigent, rural Appalachian patients. Arch. Intern. Med., 155: 421-4, 1995.

3. FONSECA, L. A. M. \& LAURENTI, R. Evolução da mortalidade materna. In: Monteiro, C. A. org. Velhos e novos males da saúde no Brasil: a evolução do País e de suas doenças. São Paulo, Hucitec / NUPENS-USP, 1995. p.186-92.

4. GERGEN, P. J.; MCQUILLAN, G. M.; KIELY, M.; EZZATI - RICE, T.M.; SUTTER, R.W.; VIRELLA, G. A populationbased serologic survey of immunity to tetanus in the United States. N. Engl. J. Med., 332: 761-6, 1995

5. JOLLIET, P.; MAGNENAT, J. L.; KOBEL, T.; CHEVROLET, J. C. Aggressive intensive care treatment of very elderly patients with tetanus is justified. Chest, 97: 7025,1990 .

6. KNIGHT, A. L. \& RICHARDSON, J. P. Management of tetanus in the elderly. J. Am. Board Fam. Pract., 5: 43-9, 1992.

7. LAFORCE, F. M. Control of neonatal tetanus by maternal immunization: an international success story. In: Interscience Conference on Antimicrobial Agents and Chemotherapy, 36th, New Orleans, 1996. Abstracts-On-Disk [S046], 1996.

8. LITVOC, J.; LEITE, R. M.; KATZ, G. Aspectos epidemiológicos do tétano no Estado de São Paulo (Brasil). Rev. Inst. Med. Trop. S. Paulo, 33: 477-84, 1991.

9. LUISTO, M. \& SEPPALAINEN, A. M. Tetanus caused by occupational accidents. Scand. J. Work Environ. Health, 18: 323-6, 1992.
10. MINISTÉRIO DA SAÚDE. Fundação Nacional da Saúde. Centro Nacional de Epidemiologia Séries históricas de agravos e doenças transmissíveis. Inf. Epidemiol. SUS, 1:17$72,1992$.

11. MINISTÉRIO DA SAÚDE. Fundação Nacional da Saúde. Centro Nacional de Eepidemiologia. Casos de agravos e doenças infecciosas e parasitárias notificados em $1994 \mathrm{e}$ 1995, por unidade federada, Brasil. Tétano. Inf. Epidemiol. SUS, 4:101-24, 1995.

12. OLSEN, A. D.; BISTRUP, M. L.; BJERREGAARD, P. Vaccinationsstatus blandt born ved skolestart 1988/1989. Ugeskr Laeger., 156: 7504-10, 1994.

13. ONER, C. Le curare dans le traitement du tetanos. Cah. Anesthesiol. 38: 355-9, 1990.

14. SABROSA, P. C.; KAWA, H.; CAMPOS, W. S. Q. Doenças transmissíveis: ainda um desafio. In: Minayo, M. C. S. org. Os muitos Brasis: saúde e população na década de 80. São Paulo, HUCITEC/ABRASCO, 1995. p. 177-244.

15. SANFORD, J. P. Tetanus-forgotten but not gone. N. Engl. J. Med., 332: 812-3, 1995.

16. STEIN, B. E. Vaccinating elderly people: protecting from avoidable disease. Drugs Aging., 5: 242-53, 1994.

17. VACHON, F. Tetanos. Epidemiologie, etiologie, physiopathologie, diagnostic, evolution et pronostic, principes du traitement curatif, preventif et prophylactique. Rev. Prat., 40: 1791-3, 1990.

18. WALDMAN, E. A.; SILVA, L. J.; MONTEIRO, C. A. Trajetória das doenças infecciosas: da eliminação da poliomielite à reintrodução da cólera. In: Monteiro, C. A., org. Velhos e novos males da saúde no Brasil: a evolução do País e de suas doenças. São Paulo, Hucitec / NUPENS-USP, 1995. p.195-244. 